\title{
Importance of Quantum Tunneling in Vacancy-Hydrogen Complexes in Diamond
}

\author{
M. J. Shaw, P. R. Briddon, J. P. Goss, and M. J. Rayson \\ School of Natural Science (Physics) The University of Newcastle upon Tyne, Newcastle upon Tyne, NE1 7RU, United Kingdom
}

A. Kerridge, A. H. Harker, and A. M. Stoneham

Department of Physics and Astronomy, University College London, Gower Street, London WC1E 6BT. United Kingdom

(Received 16 March 2005; published 2 September 2005)

\begin{abstract}
Our $a b$ initio calculations of the hyperfine parameters for negatively charged vacancy-hydrogen and nitrogen-vacancy-hydrogen complexes in diamond compare static defect models and models which account for the quantum tunneling behavior of hydrogen. The static models give rise to hyperfine splittings that are inconsistent with the experimental electron paramagnetic resonance data. In contrast, the hyperfine parameters for the quantum dynamical models are in agreement with the experimental observations. We show that the quantum motion of the proton is crucial to the prediction of symmetry and hyperfine constants for two simple defect centers in diamond. Static a priori methods fail for these systems.
\end{abstract}

PACS numbers: 61.72.Bb, 61.72.Ji, 66.35.+a, 76.30.Mi

The identification and characterization of defects in semiconductors is critical to the continued engineering of high-quality materials for device applications. With the development of high-quality single crystal chemical vapor deposition (CVD) diamond, exhibiting properties potentially suitable for semiconductor applications [1], the role of defects in determining electronic and optical properties is becoming increasingly relevant. One element which has become the focus of considerable attention is hydrogen [2], typically present in the CVD growth process, which can be incorporated into the layer. The first step towards understanding and potentially manipulating hydrogen defects is a microscopic model of the hydrogen defects in the lattice. In particular, hydrogen has been identified in complexes with other contaminant defects (e.g., nitrogen) and native defects (e.g., vacancies). For example, the nitrogenvacancy-hydrogen $(\mathrm{N}-\mathrm{V}-\mathrm{H})$ complex has been reported and investigated, leading to proposed models of the microscopic structure of the complex [3,4].

For defect complexes containing hydrogen atoms there has been some debate about the role of the quantum mechanical behavior of the hydrogen atoms. This raises the possibility that conventional density-functional theory (DFT) calculations, in which the hydrogen atoms are regarded as static, may in general be misleading and lead to spurious conclusions. Here we study the particular case of the hyperfine parameters of hydrogen defects in diamond to demonstrate the importance of hydrogen tunneling in determining the qualitative and quantitative characteristics of the defects. The implications of our results, however, reach beyond the specific examples chosen, and are of general importance to the study of hydrogen-containing defects in solids, and indeed to more general systems containing hydrogen.

In a recent paper, Kerridge et al. [5] described a method for the rapid evaluation of the quantum wave function of a light interstitial (typically a proton or muon) in a variety of defect complexes. This method has been implemented so that self-consistent calculations of zero-point motion can be made in a time comparable to standard self-consistentfield approximation calculations. Results for the present systems reveal that the quantum nature of the interstitial must be taken into account in analysis of the defect complex. Our approach makes use of a parametrized model potential that accounts for the dynamical correlation of the electrons to the motion of the interstitial, a critical feature in the quantitative analysis of these defect systems. It is this correlation which gives the interstitial $\mathrm{H}$ its delocalized nature, crucial for complexes where the inclusion of zero-point motion and nonzero tunneling probabilities can qualitatively alter the observed defect character, either through tunneling between equivalent sites in the complex, or through classical hopping via an effective barrier reduced by the zero-point energy. Such dynamical features of the complex are overlooked if the interstitial is treated classically.

This new method has been used to study the negatively charged $\mathrm{N}-V-\mathrm{H}^{-}$defect complex in diamond. The experimental electron paramagnetic resonance (EPR) data indicates a defect complex with $C_{3 v}$ symmetry, and a model in which the $\mathrm{H}$ bonds to the $\mathrm{N}$ atom has been proposed [3]. However, $a b$ initio theory in which the $\mathrm{H}$ nuclei are treated classically predicts an equilibrium structure in which the $\mathrm{H}$ bonds to one of the three unsaturated $\mathrm{C}$ atoms at the vacancy [4], giving a defect of $C_{1 h}$ symmetry in clear contradiction with experiment. Quantum tunneling of the $\mathrm{H}$ between the three equivalent $\mathrm{C}$ sites could account for the observed symmetry-calculations of the tunneling time [5] indicate that the EPR measurement would indeed average the contributions from the three sites. Figure 1 shows the static nitrogen-bonded model of Ref. [3] and dynamic models for the $\mathrm{N}-V-\mathrm{H}^{-}$defect. 
a) Static NVH defect

b) Dynamic NVH defect

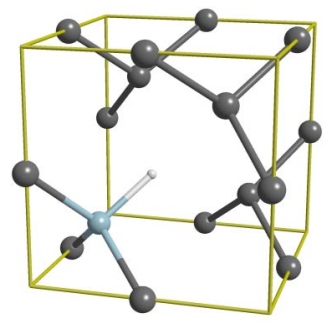

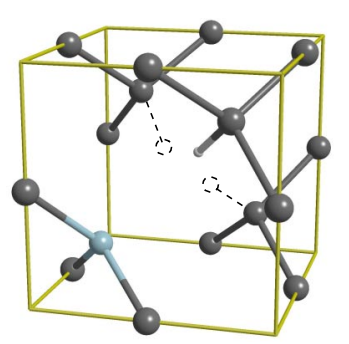

a) VH defect

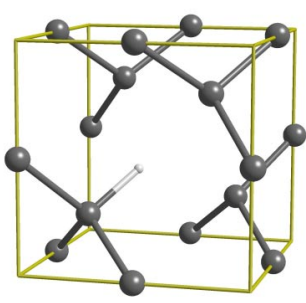

b) $\mathrm{V}_{2} \mathrm{H}$ defect

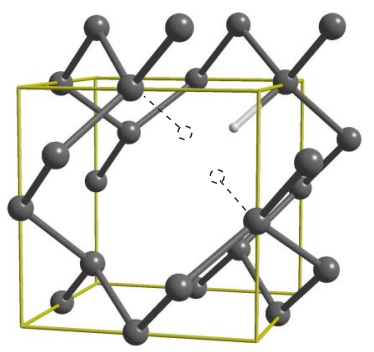

FIG. 1 (color online). (a) the static, and (b) the dynamic, $\mathrm{N}-V-\mathrm{H}^{-}$defect model. Dark gray atoms represent $\mathrm{C}$, light gray $\mathrm{N}$, and the small white atom is the H. For (b) the atoms are shown at equilibrium for one of the three equivalent $\mathrm{H}-\mathrm{C}$ bonding sites. The dashed lines indicate the other tunneling sites.

Similarly, in a recent paper, Glover et al. [6] identified a negatively charged vacancy-hydrogen $\left(V-\mathrm{H}^{-}\right)$defect with EPR data. In order to explain the observed experimental data they propose a model for the defect complex of a hydrogen atom bonded to one of the $4 \mathrm{C}$ atoms at the vacancy, justifying the observed hyperfine splittings using a simple point dipole calculation. An alternative model for this data exists in the negatively charged divacancyhydrogen complex $\left(V_{2}-\mathrm{H}^{-}\right)$- the hydrogen tunnels between three equivalent $\mathrm{C}$ sites neighboring a negatively charged divacancy. We have performed calculations using the parametrized correlation model of Ref. [5] on two diamond clusters, one containing a single $V_{2}-\mathrm{H}^{-}$complex and the other a single $V-\mathrm{H}^{-}$complex. These calculations yield a tunnel splitting energy and tunneling period of $0.1 \mathrm{~cm}^{-1}$ and $320 \mathrm{ps}$ for $V_{2}-\mathrm{H}^{-}$, respectively, and $0.6 \mathrm{~cm}^{-1}$ and $50 \mathrm{ps}$ for $V-\mathrm{H}^{-}$. While these tunneling periods are longer than that calculated for the $\mathrm{N}-\mathrm{V}-\mathrm{H}^{-}$ complex, and are comparable to typical EPR time resolution, there is good reason to believe that these values should be taken as upper limits on the period, and so dynamic models are viable [7]. The two structures are shown in Fig. 2. A $V_{2}-\mathrm{H}^{-}$complex was assigned by Iakoubovskii and Stesmans [8] to the KUL2 EPR spectra, although they do not speculate as to its microscopic structure. Inclusion of quantum tunneling for the $\mathrm{H}$ provides an alternative model displaying the observed EPR symmetry.

Hyperfine parameters obtained from EPR and electronnuclear double resonance technique spectroscopy are one of the key sources of experimental data on defect complexes. The challenge for theory is to obtain reliable calculations of the hyperfine parameters for a specific microscopic defect model to compare with experimental data. However, the calculation of hyperfine parameters for defects in semiconductors is particularly demanding - the theory must provide a detailed description of the chemical bonding around the defect structure, and also accurately describe the very rapid charge density fluctuations which occur in the close vicinity of the nuclei. Here we employ a theoretical scheme, based upon ab initio pseudopotential
FIG. 2 (color online). (a) the static $V$ - $\mathrm{H}^{-}$defect, and (b) the dynamic $V_{2}-\mathrm{H}^{-}$defect. Gray atoms represent $\mathrm{C}$, while the small white atom is the $\mathrm{H}$. In (b) the atoms are shown at equilibrium for one of the three equivalent $\mathrm{H}-\mathrm{C}$ bonding sites. The dashed lines indicate the configurations between which tunneling occurs.

calculations with all-electron wave function reconstruction, which achieves each of these for moderate computational expense. With this theoretical procedure, alternative defect models can be distinguished according to their hyperfine couplings.

The theoretical method for our calculations of hyperfine splittings is outlined briefly below, and will be described in full elsewhere in the literature. A conventional $a b$ initio pseudopotential model is combined with a reconstruction of all-electron wave functions in the core region $[9,10]$. Dual-space separable first-principles pseudopotentials [11] are applied in the local spin-density approximation of DFT to obtain the pseudowave functions for the defect system. A Gaussian orbital basis set is used with the AIMPRO code [12], for defect unit cells based upon the 64 atom cubic cell of bulk diamond. The pseudowave functions obtained in this way provide an accurate representation of the chemical bonding in the defect, but they are smooth close to the nuclei by construction. However, the rapid wave function variations in the region of the ion cores can be modeled by reconstruction using all-electron wave functions obtained for isolated atoms.

The analysis of EPR experimental data yields values of the hyperfine tensor, $\underline{\mathbf{A}}$, which couples the electronic spin, $\mathbf{S}$, to the nuclear spin, $\mathbf{I}$ through terms in the spin Hamiltonian $\mathbf{S} \cdot \underline{\mathbf{A}} \cdot \mathbf{I}$. The hyperfine matrix describes two effects: the Fermi contact term arising from the nonzero spin density at the nucleus, and the dipole-dipole interaction. Both of these depend upon the form of the electronic wave functions within the ion core region. Reconstruction allows us to calculate the hyperfine tensor elements with frozen-core all-electron wave functions, without the computational difficulties associated with a full all-electron calculation.

The static $\mathrm{N}-V-\mathrm{H}^{-}$defect proposed by Glover et al. [3] has the $\mathrm{H}$ atom bonded to the $\mathrm{N}$ with a bond length of $1.0 \AA$. One of the difficulties with this model is that one would not expect the $\mathrm{H}$ and $\mathrm{N}$ to form a bond at all in these circumstances. Indeed, our DFT calculations confirm this, 
and relaxing the positions of all of the atoms (with the $\mathrm{H}$ constrained to lie on the [111] axis), the minimum energy occurs with a H-N distance of $3.8 \AA$, clearly unbonded. The hydrogen hyperfine parameters for this minimum energy structure, and for the bond length of Ref. [3] are compared to experiment in Table I.

To calculate the hyperfine parameters for the dynamical $\mathrm{N}-V-\mathrm{H}^{-}$model we begin with a static defect in which the $\mathrm{H}$ is bonded to one of the unsaturated $\mathrm{C}$ atoms. With the symmetry so defined, the positions of all atoms were fully relaxed. Contributions to the hyperfine tensor must be averaged over the probability distribution for the position of the hydrogen nucleus. To a first approximation we can model this distribution to that of a vibrational mode of the $\mathrm{C}-\mathrm{H}$ bond, and then average over the three equivalent $\mathrm{C}-\mathrm{H}$ bonding sites. The probability density in the zero-point vibrational mode is estimated from phonon frequencies calculated using the AIMPRO code. The hyperfine parameters computed in this way are compared to a simple averaging over the three equivalent minimum energy positions in Table I. Clearly, the correction due to the distribution over the vibrational mode is relatively small, and we therefore predict that further improving the model to account for the 3D motion of the proton will make only minor changes to the hyperfine parameters.

It is clear from Table I that the hyperfine parameters of the dynamical model are in good agreement with the cited EPR data. In contrast, the N-H bonded model does not agree with experiment for either of the $\mathrm{N}-\mathrm{H}$ bond lengths presented. Indeed further study shows that there is no N-H bond length for which the result agrees with experiment. Of course, if the $\mathrm{N}-\mathrm{H}$ bonding model is correct then it is clear that the DFT calculation upon which our analysis is based must be breaking down - it categorically predicts that the N-H bond structure will be unstable and so the hyperfine parameters of that structure must be treated with caution. We can conclude, however, that the dynamical defect is not only feasible according to the quantum mechanical treatment of the hydrogen nucleus, but also that its hyperfine parameters are in good agreement with the experiment. Accounting for $\mathrm{H}$ tunneling, the lowest energy

TABLE I. The hyperfine parameters $(\mathrm{MHz})$ for the $\mathrm{N}-V-\mathrm{H}^{-}$ defect. $\mathrm{A}_{\|}$and $\mathrm{A}_{\perp}$ are given where $\|$ refers to the [111] direction. "Bond" refers to the bond formed by the $\mathrm{H}$ and, for $\mathrm{N}-\mathrm{H}$ bonding to the bond length (optimum or from Ref. [3]), or for $\mathrm{C}-\mathrm{H}$ to the averaging (over minimum energy sites only or phonon distribution also). Experimental values of Ref. [6] are listed.

\begin{tabular}{llcc}
\hline \hline \multicolumn{1}{c}{ Model } & \multicolumn{1}{c}{ Bond } & $\mathrm{A}_{\|}(\mathrm{MHz})$ & $\mathrm{A}_{\perp}(\mathrm{MHz})$ \\
\hline Static & $\mathrm{N}-\mathrm{H}$ (minimum) & -64.2 & -15.3 \\
Static & $\mathrm{N}-\mathrm{H}$ (Glover) & -4.4 & -1.9 \\
Dynamic & $\mathrm{C}-\mathrm{H}$ (minimum) & -12.2 & 10.6 \\
Dynamic & $\mathrm{C}-\mathrm{H}$ (phonon) & -12.6 & 10.2 \\
Experiment & & \pm 13.7 & \pm 9.0 \\
\hline \hline
\end{tabular}

structure predicted by theory yields hyperfine parameters consistent with the symmetry and values identified from experiment.

The $V-\mathrm{H}^{-}$defect proposed in Ref. [6] was also modeled, relaxing the positions of all of the atoms in the unit cell to obtain the minimum energy structure. The minimum energy configuration has a C-H bond length of $1.09 \AA$, consistent with the $1.0 \pm 0.1 \AA$ of Ref. [6]. The hyperfine parameters for the hydrogen atom in this defect complex are presented in Table II. Experimental data is included from Refs. [6,8]. The results in Table II show quite clearly that the static $V-\mathrm{H}^{-}$model is inconsistent with the experimental values. The anisotropic component of the hyperfine tensor, given by $A_{\text {aniso }}=\left(A_{\|}-A_{\perp}\right) / 3$, is $-45 \mathrm{MHz}$ while the maximum anisotropic component from experiment is $1 \mathrm{MHz}$. This provides strong evidence that the $V-\mathrm{H}^{-}$ complex is not the defect which is responsible for the EPR center.

An alternative model for a defect with $C_{3 v}$ symmetry would be the dynamic model of a hydrogen atom tunneling between the $3 \mathrm{C}$ sites at one end of a divacancy [Fig. 2(b)]. This is modeled by first obtaining the epitomized structure for the static complex in which the $\mathrm{H}$ is bonded to one of the $\mathrm{C}$ atoms. Now, for the dynamic $V-\mathrm{H}^{-}$defect we demonstrated that averaging over the proton distribution made very little difference to the hyperfine parameters. For the present system we therefore account for the tunneling of the $\mathrm{H}$ in the most basic way by averaging over equivalent $\mathrm{H}-\mathrm{C}$ bonded sites. The hyperfine parameters for this model are also included in Table II.

The static $V-\mathrm{H}^{-}$model clearly contradicts the experimental evidence. By contrast, the hyperfine parameters for the dynamical $V_{2}-\mathrm{H}^{-}$complex are in order of magnitude agreement with the experimental values, and in agreement to within the accuracy of our calculations for such small hyperfine splittings. For hyperfine parameters as small as this the accuracy of our hyperfine calculations do not allow us to positively assign the $V_{2}-\mathrm{H}^{-}$complex to either of the experiments.

The tunneling of hydrogen is not restricted to the particular systems we have studied. For example, the H1 and H2 EPR centers have been linked with similar systems and may have similar motional effects to be taken into account in order that a correct microscopic assignment can be made [13]. In particular, $\mathrm{H} 1$ has been given a near-trigonal

TABLE II. The hyperfine parameters $(\mathrm{MHz})$ for the $V-\mathrm{H}^{-}$and dynamical $V_{2}-\mathrm{H}^{-}$complexes. $\mathrm{A}_{\|}$and $\mathrm{A}_{\perp}$ are given where $\|$ refers to the [111] direction. The experimental values of Refs. $[6,8]$ are listed.

\begin{tabular}{lcc}
\hline \hline \multicolumn{1}{c}{ System } & $\mathrm{A}_{\|}(\mathrm{MHz})$ & $\mathrm{A}_{\perp}(\mathrm{MHz})$ \\
\hline Static $V-\mathrm{H}^{-}$ & -86.6 & 48.1 \\
Dynamic $V_{2}-\mathrm{H}^{-}$ & 2.4 & 0.8 \\
Expt. Ref. [6] & \pm 1.1 & \pm 2.0 \\
Expt. Ref. [8] & \pm 2.8 & \pm 1.1 \\
\hline \hline
\end{tabular}


symmetry [14]. It has also been suggested that these centers are not simple hydrogen-vacancy centers, but are instead associated with $\mathrm{H}$ trapped at an extended defect of lower symmetry [15]. It could be that inclusion of dynamical effects would resolve the apparent contradiction. Our present work indicates that such effects should either be included or be explicitly ruled out if either quantum mechanical calculations or the symmetry of EPR measurements are to be used as tools in the assignment of hydrogen-containing defects.

Our N-V-H system in diamond shows competition between the tunneling of an atom and simple energetics, and is striking because quantum behavior affects the observed symmetry. Could this happen more generally? The answer is surely yes. Our methods can be applied to estimate the key matrix elements for the incoherent tunneling processes often seen, e.g., for $\mathrm{H}$ motion associated with $\mathrm{Si}: \mathrm{B}$ [16], $\mathrm{Si}: \mathrm{Cd}$ [17], Nb:N [18] and $\mathrm{Nb}: \mathrm{O}$ [19]. In $\mathrm{Si}, \mathrm{H}$ shows incoherent tunneling around substitutional $\mathrm{B}$ and $\mathrm{Cd}$ [16]. For $\mathrm{Si}$ :B the muon, however, does not show the quadrupole resonance expected if it occupied the same site as the heavier hydrogen species [20], but is consistent with the higher symmetry expected from tunneling, as we have described. But such quantum behavior need not be confined to simple structures. In bioscience, proton tunneling is common [21] and some structures will surely involve competition between static energies and quantum tunneling. Another possible example concerns the very complex phase diagram of ice $[22,23]$. Discussions usually ignore quantum $\mathrm{H}$, yet it is possible (if unproven) that some of the apparent complexity stems from the competition we describe.

In conclusion, we have presented calculations of the hyperfine parameters for model hydrogen-containing defects in which quantum tunneling effects have been included. For the $\mathrm{N}-V-\mathrm{H}^{-}$defect we have shown that the static model of Glover et al. does not result in hyperfine parameters consistent with experiment, while a static model of the DFT equilibrium structure has a lower symmetry than seen experimentally. However, a model in which the $\mathrm{H}$ atom is allowed to tunnel between equivalent equilibrium sites restores the correct symmetry. Furthermore, the hyperfine parameters for this structure are in agreement with the experimental values. Similarly, we find that the hyperfine parameters of the static $V-\mathrm{H}^{-}$defect disagree with the EPR data to which they are assigned by Glover et al.. Again, a dynamical tunneling model, this time for a hydrogen divacancy complex, provides an alternative model in broad agreement with experiment.

We have demonstrated the role $a b$ initio calculations of hyperfine parameters can play in helping to distinguish between different models of defects, and propose alternative models for recently characterized centers which rely upon a quantum mechanical treatment of the hydrogen nucleus. Quantum tunneling of the hydrogen is found to be critical in identifying the correct microscopic structure for defect complexes. Importantly, this quantum tunneling makes the results of standard DFT calculations misleading, qualitatively changing the results. Furthermore, although the effect has been demonstrated for some specific defect examples, the potential significance of the quantum tunneling behavior will extend in general to other hydrogencontaining defect centers in diamond and other materials.

The authors would like to thank EPSRC for financial support. A. K. would like to thank the Rutherford Appleton Laboratories for financial support.

[1] J. Isberg, J. Hammersberg, E. Johansson, T. Wikström, D. J. Twitchen, A. J. Whitehead, S.E. Coe, and G. A. Scarsbrook, Science 297, 1670 (2002).

[2] J. P. Goss, J. Phys. Condens. Matter 15, R551 (2003).

[3] C. Glover, M.E. Newton, P. Martineau, D. J. Twitchen, and J. M. Baker, Phys. Rev. Lett. 90, 185507 (2003).

[4] J. P. Goss, P. R. Briddon, R. Jones, and S. Sque, J. Phys. Condens. Matter 15, R2903 (2003).

[5] A. Kerridge, A. H. Harker, and A. M. Stoneham, J. Phys. Condens. Matter 16, 8743 (2004).

[6] C. Glover, M. E. Newton, P. M. Martineau, S. Quinn, and D. J. Twitchen, Phys. Rev. Lett. 92, 135502 (2004).

[7] A. Kerridge, Ph.D. thesis, University of London, UK, 2004.

[8] K. Iakoubovskii and A. Stesmans, Phys. Rev. B 66, 195207 (2002).

[9] P. E. Blöchl, Phys. Rev. B 50, 17953 (1994).

[10] B. Hetényi, F. De Angelis, P. Giannozzi, and R. Car, J. Chem. Phys. 115, 5791 (2001).

[11] C. Hartwigsen, S. Goedecker, and J. Hutter, Phys. Rev. B 58, 3641 (1998).

[12] P. R. Briddon and R. Jones, Phys. Status Solidi B 217, 131 (2000).

[13] X. Zhou, G. D. Watkins, K. M. McNamara Rutledge, R. P. Messner, and S. Chawla, Phys. Rev. B 54, 7881 (1996).

[14] K. Iakoubovskii, A. Stesmans, K. Suzuki, A. Sawabe, and T. Yamada, Phys. Rev. B 66, 113203 (2002).

[15] D. F. Talbot-Ponsonby, M. E. Newton, J. M. Baker, G. A. Scarsbrook, R. S. Sussmann, and A.J. Whitehead, Phys. Rev. B 57, 2302 (1998).

[16] A. M. Stoneham, Phys. Rev. Lett. 63, 1027 (1989).

[17] M. Gebhard, B. Vogt, and W. Witthuhn, Phys. Rev. Lett. 67, 847 (1991).

[18] C. G. Chen and H. K. Birnbaum, Acta Metall. 28, 1523 (1980).

[19] C. G. Chen and H. K. Birnbaum, Phys. Status Solidi A 36, 687 (1976).

[20] D. M. Maric, P. F. Meier, S. Vogel, S. F. J. Cox, E. A. Davis, and J. W. Schneider, J. Phys. Condens. Matter 3, 9675 (1991).

[21] M. J. Sutcliffe and N. S. Scrutton, Phil. Trans. R. Soc. A 358, 367 (2000).

[22] J. Finney, C. Lobban, and W.F. Kuhs, Nature (London) 391, 268 (1998).

[23] D. D. Klug, Nature (London) 420, 749 (2002). 